\title{
Analysis of Caputo Impulsive Fractional Order Differential Equations with Applications
}

\author{
Lakshman Mahto, ${ }^{1}$ Syed Abbas, ${ }^{1}$ and Angelo Favini ${ }^{2}$ \\ ${ }^{1}$ School of Basic Sciences, Indian Institute of Technology Mandi, Mandi, Himachal Pradesh 175001, India \\ ${ }^{2}$ Dipartimento di Matematica, Universitá di Bologna, Pizza di Porta S. Donato 5, 40126 Bologna, Italy \\ Correspondence should be addressed to Syed Abbas; sabbas.iitk@gmail.com
}

Received 6 May 2012; Accepted 21 November 2012

Academic Editor: Nikolai Leonenko

Copyright (C) 2013 Lakshman Mahto et al. This is an open access article distributed under the Creative Commons Attribution License, which permits unrestricted use, distribution, and reproduction in any medium, provided the original work is properly cited.

We use Sadovskii's fixed point method to investigate the existence and uniqueness of solutions of Caputo impulsive fractional differential equations of order $\alpha \in(0,1)$ with one example of impulsive logistic model and few other examples as well. We also discuss Caputo impulsive fractional differential equations with finite delay. The results proven are new and compliment the existing one.

\section{Introduction}

Dynamics of many evolutionary processes from various fields such as population dynamics, control theory, physics, biology, and medicine. undergo abrupt changes at certain moments of time like earthquake, harvesting, shock, and so forth. These perturbations can be well approximated as instantaneous change of states or impulses. These processes are modeled by impulsive differential equations. In 1960, Milman and Myšhkis introduced impulsive differential equations in their paper [1]. Based on their work, several monographs have been published by many authors like Samoilenko and Perestyuk [2], Lakshmikantham et al. [3], Bainov and Simeonov [4, 5], Bainov and Covachev [6], and Benchohra et al. [7]. All the authors mentioned perviously have considered impulsive differential equations as ordinary differential equations coupled with impulsive effects. They considered the impulsive effects as difference equations being satisfied at impulses time. So, the solutions are piecewise continuous with discontinuities at impulses time. In the fields like biology, population dynamics, and so forth, problems with hereditary are best modeled by delay differential equations [8]. Problems associated with impulsive effects and hereditary property are modeled by impulsive delay differential equations.
The origin of fractional calculus (derivatives $\left(d^{\alpha} / d t^{\alpha}\right) f$ and integrals $I^{\alpha} f$ of arbitrary order $\alpha>0$ ) goes back to Newton and Leibniz in the 17th century. In a letter correspondence with Leibniz, L'Hospital asked "What if the order of the derivative is $1 / 2$ "? Leibniz replied, "Thus it follows that will be equal to $x \sqrt{d x: x}$, an apparent paradox, from which one day useful consequences will be drawn." This letter of Leibniz was in September 1695. So, 1695 is considered as the birthday of fractional calculus. Fractional order differential equations are generalizations of classical integer order differential equations and are increasingly used to model problems in fluid dynamics, finance, and other areas of application. Recent investigations have shown that sometimes physical systems can be modeled more accurately using fractional derivative formulations [9]. There are several excellent monographs available on this field [10-15]. In [11], the authors give a recent and up-to-date description of the developments of fractional differential and fractional integrodifferential equations including applications. The existence and uniqueness of solutions to fractional differential equations has been considered by many authors [16-21]. Impulsive fractional differential equations represent a real framework for mathematical modeling to real world problems. Significant progress has been made in the theory of impulsive 
fractional differential equations [7, 22-24]. Xu et al. in their paper [25] have described an impulsive delay fishing model.

Fractional derivatives arise naturally in mathematical problems, for $\alpha>0$ and a function $f:[0, T] \rightarrow \mathbb{R}$, recall [10, Definitions 3.1, 2.2]

(a) the Caputo fractional derivative

$$
{ }^{C} D^{\alpha} f(t)=\frac{1}{\Gamma(1-\alpha)} \int_{0}^{t}(t-s)^{-\alpha} f^{\prime}(s) d s
$$

(b) the Riemann-Liouville fractional derivative

$$
D^{\alpha} f(t)=\frac{d^{\alpha}}{d t^{\alpha}} f(t)=\frac{1}{\Gamma(1-\alpha)} \frac{d}{d t} \int_{0}^{t}(t-s)^{-\alpha} f(s) d s,
$$

provided that the right-hand sides exist pointwise on $[0, T](\Gamma$ denotes the gamma function). Using the Riemann-Liouville fractional integral [10, Definition 2.1] $I_{0}^{\alpha} f(t)=(1 / \Gamma(\alpha)) \int_{0}^{t}(t-$ $s)^{\alpha-1} f(s) d s$, we have ${ }^{C} D^{\alpha} f(t)=I_{0}^{1-\alpha}(d / d t) f(t)$ and $D^{\alpha} f(t)=(d / d t) I_{0}^{1-\alpha} f(t) . I_{0}^{\alpha} f$ exists, for instance, for all $\alpha>0$, if $f \in C^{0}([0, T]) \cap L_{\text {loc }}^{1}([0, T])$; moreover, $I_{0}^{\alpha} f(0)=0$. Throughout the paper, we assume that $I=[0, T]$.

One can see that both of the fractional derivatives are actually nonlocal operator because integral is a nonlocal operator. Moreover, calculating time fractional derivative of a function at some time requires all the past history, and hence fractional derivatives can be used for modeling systems with memory. Fractional differential equations can be formulated using both Caputo and Riemann-Liouville fractional derivatives. A Riemann-Liouville initial value problem can be stated as follows:

$$
\begin{gathered}
D^{\alpha} x(t)=f(t, x(t)), \quad t \in I=[0, T], \\
D^{\alpha-1} x(0)=x_{0},
\end{gathered}
$$

or equivalently, $x(t)=x_{0} t^{\alpha-1} / \Gamma(\alpha)+\int_{0}^{t}(t-s)^{\alpha-1} f(s, x(s)) d s$ in its integral representation [11, Theorem 3.24]. For a physical interpretation of the initial conditions in (3), see [26-28]. If derivatives of Caputo type are used instead of RiemannLiouville type, then initial conditions for the corresponding Caputo fractional differential equations can be formulated as for classical ordinary differential equations, namely, $x(0)=$ $x_{0}$.

Our main objective is to discuss existence and uniqueness of solutions of the following impulsive fractional differential equation of Caputo type in a Banach space $X$ with norm $\|\cdot\|_{X}$ :

$$
\begin{gathered}
{ }^{C} D^{\alpha} x(t)=f(t, x(t)), \quad t \in I=[0, T], t \neq t_{k}, \\
\left.\Delta x(t)\right|_{t=t_{k}}=I_{k}\left(x\left(t_{k}^{-}\right)\right), \quad k=1,2, \ldots, m, \\
x(0)=x_{0},
\end{gathered}
$$

where $f \in C(I \times X, X), I_{k}: X \rightarrow X$ and $x_{0} \in X .0=t_{0}<$ $t_{1}<t_{2}<\cdots<t_{m}<t_{m+1}=T,\left.\Delta x(t)\right|_{t=t_{k}}=x\left(t_{k}^{+}\right)-x\left(t_{K}^{-}\right)$, $x\left(t_{k}^{+}\right)=\lim _{h \rightarrow 0} x(t+h)$, and $x\left(t_{k}^{-}\right)=\lim _{h \rightarrow 0} x(t-h)$. We break our function $f$ into two components which satisfy different conditions. We observed that this kind of functions occurs in ecological modeling. We have given the example of logistic equation in the last section. Our main tool is Sadovskii's fixed point theorem.

Now, we define some important spaces and norm which will encounter frequently:

$$
\begin{gathered}
\operatorname{PC}(I, X)=\left\{x:[0, T] \longrightarrow X \mid x \in C\left(\left[t_{0}, t_{1}\right], X\right)\right. \\
\cup C\left(\left(t_{k}, t_{k+1}\right], X\right) \quad k=1,2, \ldots, m, \\
\left.x\left(t_{k}^{+}\right) \text {and } x\left(t_{k}^{-}\right) \text {exist, } x\left(t_{k}\right)=x\left(t_{k}^{-}\right)\right\},
\end{gathered}
$$

with sup-norm $\|\cdot\|$, defined by $\|x\|=\sup \left\{\|x(t)\|_{X}: t \in I\right\}$.

Definition 1. A solution of fractional differential equation (4) is a piecewise continuous function $x \in \mathrm{PC}([0, T], X)$ which satisfied (4).

Definition 2 ([29], Definition 11.1). Kuratowskii noncompactness measure: let $M$ be a bounded set in metric space $(X, d)$, then Kuratowskii noncompactness measure, $\mu(M)$ is defined as $\inf \{\epsilon: M$ covered by a finite many sets such that the diameter of each set $\leq \epsilon\}$.

Definition 3 ([29], Definition 11.6). Condensing map: Let $\Phi$ : $X \rightarrow X$ be a bounded and continuous operator on Banach space $X$ such that $\mu(\Phi(B))<\mu(B)$ for all bounded set $B \subset$ $D(\Phi)$, where $\mu$ is the Kuratowskii noncompactness measure, then $\Phi$ is called condensing map.

Definition 4. Compact map: a map $f: X \rightarrow X$ is said to be compact if the image of every bounded subset of $X$ under $f$ is precompact (closure is compact).

Theorem 5 (see [30]). Let B be a convex, bounded, and closed subset of a Banach space $X$ and let $\Phi: B \rightarrow B$ be a condensing map. Then, $\Phi$ has a fixed point in $B$.

Lemma 6 ([29], Example 11.7). A map $\Phi=\Phi_{1}+\Phi_{2}: X \rightarrow X$ is $k$-contraction with $0 \leq k<1$ if

(a) $\Phi_{1}$ is $k$-contraction, that is, $\left\|\Phi_{1}(x)-\Phi_{1}(y)\right\|_{X} \leq$ $k\|x-y\|_{X}$;

(b) $\Phi_{2}$ is compact,

and hence $\Phi$ is a condensing map.

The structure of the paper is as follows. In Section 2, we prove the existence (Theorem 8) and uniqueness of solutions to (4). We show in Section 3 the existence and uniqueness of solutions for a general class of impulsive functional differential equations of fractional order $\alpha \in(0,1)$. In Section 4 , we give some examples in favor of our sufficient conditions. 


\section{Impulsive Fractional Differential Equation}

Consider the initial value problem (4) on the cylinder $R=$ $\{(t, x) \in \mathbb{R} \times X: t \in[0, T], x \in B(0, r)\}$ for some fixed $T>0$, $r>0$, and assume that there exist $p \in(0, \alpha), \alpha \in(0,1)$, $M_{1}, M_{2}, L_{1} \in L_{1 / p}\left([0, T], \mathbb{R}^{+}\right)$and functions $f_{1}, f_{2} \in C(R \times$ $X, X)$ such that $f=f_{1}+f_{2}$, and the following assumptions are satisfied:

(A.1) $f_{1}$ is bounded and Lipschitz, in particular, $\left\|f_{1}(t, x)\right\|_{X} \leq M_{1}(t)$ and $\left\|f_{1}(t, x)-f_{1}(t, y)\right\|_{X} \leq$ $L_{1}(t)\|x-y\|_{X}$ for all $(t, x),(t, y) \in R$,

(A.2) $f_{2}$ is compact and bounded, in particular, $\left\|f_{2}(t, x)\right\|_{X} \leq M_{2}(t)$ for all $(t, x) \in R$,

(A.3) $I_{k} \in C(X, X)$ such that $\left\|I_{k}(x)\right\|_{X} \leq l_{1}$ and $\| I_{k}(x)-$ $I_{k}(y)\left\|_{X} \leq l_{2}\right\| x-y \|_{X}$,

where $C(R \times X, X)$ denotes set of continuous functions from $R \times X$ to $X$ and $L_{1 / p}\left([0, T], \mathbb{R}^{+}\right)$denotes space $1 / p$-Lesbegue measurable functions from $[0, T]$ to $\mathbb{R}^{+}$with norm $\|\cdot\|_{1 / p}$.

Lemma 7 (Fečken et al. [24], Lemma 2). The initial value problem (4) is equivalent to the nonlinear integral equation

$$
\begin{aligned}
x(t)= & x_{0}+\frac{1}{\Gamma(\alpha)} \int_{0}^{t}(t-s)^{\alpha-1} f_{1}(s, x(s)) d s \\
& +\frac{1}{\Gamma(\alpha)} \int_{0}^{t}(t-s)^{\alpha-1} f_{2}(s, x(s)) d s, \quad t \in\left[0, t_{1}\right] \\
= & x_{0}+I_{1}\left(x\left(t_{1}^{-}\right)\right)+\frac{1}{\Gamma(\alpha)} \int_{0}^{t}(t-s)^{\alpha-1} f_{1}(s, x(s)) d s \\
& +\frac{1}{\Gamma(\alpha)} \int_{0}^{t}(t-s)^{\alpha-1} f_{2}(s, x(s)) d s, \quad t \in\left(t_{1}, t_{2}\right] \\
= & x_{0}+\sum_{k=1}^{2} I_{k}\left(x\left(t_{k}^{-}\right)\right)+\frac{1}{\Gamma(\alpha)} \int_{0}^{t}(t-s)^{\alpha-1} f_{1}(s, x(s)) d s \\
& +\frac{1}{\Gamma(\alpha)} \int_{0}^{t}(t-s)^{\alpha-1} f_{2}(s, x(s)) d s, \quad t \in\left(t_{2}, t_{3}\right] \\
= & x_{0}+\sum_{k=1}^{m} I_{k}\left(x\left(t_{k}^{-}\right)\right)+\frac{1}{\Gamma(\alpha)} \int_{0}^{t}(t-s)^{\alpha-1} f_{1}(s, x(s)) d s \\
& +\frac{1}{\Gamma(\alpha)} \int_{0}^{t}(t-s)^{\alpha-1} f_{2}(s, x(s)) d s, \quad t \in\left(t_{m}, T\right] .
\end{aligned}
$$

In other words, every solution of the integral equation (6) is also solution of our original initial value problem (4) and conversely.

Theorem 8 (existence of solutions). Under the assumptions (A.1)-(A.3), the problem (4) has at least one solution in $[0, T]$, provided that

$$
\gamma_{1}=m l_{2}+\frac{c\left\|L_{1}\right\|_{1 / p} T^{\alpha-p}}{\Gamma(\alpha+1)}<1, \quad \text { where } c=\left(\frac{1-p}{\alpha-p}\right)^{1-p} .
$$

Proof. Let $B_{\lambda}$ be the closed bounded and convex subset of $\operatorname{PC}([0, T], X)$, where $B_{\lambda}$ is defined as $B_{\lambda}=\{x:\|x\| \leq \lambda\}, \lambda=$ $\max \left\{\lambda_{0}, \lambda_{1}, \ldots, \lambda_{m}\right\}$, and

$$
\begin{array}{r}
\lambda_{k}=\left\|x_{0}\right\|_{X}+k l_{1}+\frac{c\left(\left\|M_{1}\right\|_{1 / p}+\left\|M_{2}\right\|_{1 / p}\right)}{\Gamma(\alpha)} T^{\alpha-p}, \\
k=0,1,2, \ldots, m .
\end{array}
$$

Define a map $F: B_{\lambda} \rightarrow X$ such that

$$
\begin{aligned}
F x(t)= & x_{0}+\sum_{0<t_{k}<t} I_{k}\left(x\left(t_{k}^{-}\right)\right) \\
& +\frac{1}{\Gamma(\alpha)} \int_{0}^{t}(t-s)^{\alpha-1} f_{1}(s, x(s)) d s \\
& +\frac{1}{\Gamma(\alpha)} \int_{0}^{t}(t-s)^{\alpha-1} f_{2}(s, x(s)) d s .
\end{aligned}
$$

Let us consider that

$$
\begin{aligned}
F_{1} x(t)= & x_{0}+\sum_{0<t_{k}<t} I_{k}\left(x\left(t_{k}^{-}\right)\right) \\
& +\frac{1}{\Gamma(\alpha)} \int_{0}^{t}(t-s)^{\alpha-1} f_{1}(s, x(s)) d s, \\
F_{2} x(t)= & \frac{1}{\Gamma(\alpha)} \int_{0}^{t}(t-s)^{\alpha-1} f_{2}(s, x(s)) d s .
\end{aligned}
$$

Step 1 ( $F$ is self mapping). Now, we show that $F\left(B_{r}\right) \subset B_{r}$. For $t \in\left[0, t_{1}\right]$

$$
\begin{aligned}
& \|F x(t)\|_{X} \leq\left\|x_{0}\right\|_{X}+\frac{1}{\Gamma(\alpha)} \int_{0}^{t}(t-s)^{\alpha-1}\|f(s \cdot x(s))\|_{X} d s \\
& \leq\left\|x_{0}\right\|_{X}+\frac{1}{\Gamma(\alpha)} \int_{0}^{t}(t-s)^{\alpha-1}\left\|f_{1}(s, x(s))\right\|_{X} d s \\
& +\frac{1}{\Gamma(\alpha)} \int_{0}^{t}(t-s)^{\alpha-1}\left\|f_{2}(s, x(s))\right\|_{X} d s \\
& \leq\left\|x_{0}\right\|_{X}+\frac{1}{\Gamma(\alpha)} \int_{0}^{t}(t-s)^{\alpha-1} M_{1}(s) d s \\
& +\frac{1}{\Gamma(\alpha)} \int_{0}^{t}(t-s)^{\alpha-1} M_{2}(s) d s \\
& \leq\left\|x_{0}\right\|_{X}+\frac{1}{\Gamma(\alpha)}\left(\int_{0}^{t}(t-s)^{(\alpha-1) /(1-p)} d s\right)^{1-p} \\
& \times\left(\int_{0}^{t} M_{1}^{1 / p}(s) d s\right)^{p}+\frac{1}{\Gamma(\alpha)} \\
& \times\left(\int_{0}^{t}(t-s)^{(\alpha-1) /(1-p)} d s\right)^{1-p}\left(\int_{0}^{t} M_{2}^{1 / p}(s) d s\right)^{p} \\
& \leq\left\|x_{0}\right\|_{X}+\frac{c\left(\left\|M_{1}\right\|_{1 / p}+\left\|M_{2}\right\|_{1 / p}\right)}{\Gamma(\alpha)} T^{\alpha-p}=\lambda_{0} \text {. }
\end{aligned}
$$


For $t \in\left(t_{1}, t_{2}\right]$,

$$
\begin{aligned}
\|F x(t)\|_{X} \leq & \left\|x_{0}\right\|_{X}+\left|I_{1}\left(x\left(t_{1}^{-}\right)\right)\right| \\
& +\frac{1}{\Gamma(\alpha)} \int_{0}^{t}(t-s)^{\alpha-1}\|f(s \cdot x(s))\|_{X} d s \\
\leq & \left\|x_{0}\right\|_{X}+l_{1}+\frac{1}{\Gamma(\alpha)} \int_{0}^{t}(t-s)^{\alpha-1}\left\|f_{1}(s, x(s))\right\|_{X} d s \\
& +\frac{1}{\Gamma(\alpha)} \int_{0}^{t}(t-s)^{\alpha-1}\left\|f_{2}(s, x(s))\right\|_{X} d s \\
\leq & \left\|x_{0}\right\|_{X}+l_{1}+\frac{1}{\Gamma(\alpha)} \int_{0}^{t}(t-s)^{\alpha-1} M_{1}(s) d s \\
& +\frac{1}{\Gamma(\alpha)} \int_{0}^{t}(t-s)^{\alpha-1} M_{2}(s) d s \\
\leq & \left\|x_{0}\right\|_{X}+l_{1}+\frac{1}{\Gamma(\alpha)} \\
& \times\left(\int_{0}^{t}(t-s)^{(\alpha-1) /(1-p)} d s\right)^{1-p}\left(\int_{0}^{t} M_{1}^{1 / p}(s) d s\right)^{p} \\
& +\frac{1}{\Gamma(\alpha)}\left(\int_{0}^{t}(t-s)^{(\alpha-1) /(1-p)} d s\right)^{1-p} \\
& \times\left(\int_{0}^{t} M_{2}^{1 / p}(s) d s\right)^{p} \\
\leq & \left\|x_{0}\right\|_{X}+l_{1}+\frac{{ }^{2}\left(\left\|M_{1}\right\|_{1 / p}+\left\|M_{2}\right\|_{1 / p}\right)}{\Gamma(\alpha)} T^{\alpha-p} \\
= & 1_{1} .
\end{aligned}
$$

For $t \in\left(t_{2}, t_{3}\right]$,

$$
\begin{aligned}
\|F x(t)\| \leq & \left\|x_{0}\right\|_{X}+\sum_{k=0}^{2}\left|I_{k}\left(x\left(t_{k}^{-}\right)\right)\right| \\
& +\frac{1}{\Gamma(\alpha)} \int_{0}^{t}(t-s)^{\alpha-1}\|f(s \cdot x(s))\|_{X} d s \\
\leq & \left\|x_{0}\right\|_{X}+2 l_{1}+\frac{1}{\Gamma(\alpha)} \int_{0}^{t}(t-s)^{\alpha-1}\left\|f_{1}(s, x(s))\right\|_{X} d s \\
& +\frac{1}{\Gamma(\alpha)} \int_{0}^{t}(t-s)^{\alpha-1}\left|f_{2}(s, x(s))\right| d s \\
\leq & \left\|x_{0}\right\|_{X}+2 l_{1}+\frac{1}{\Gamma(\alpha)} \int_{0}^{t}(t-s)^{\alpha-1} M_{1}(s) d s \\
& +\frac{1}{\Gamma(\alpha)} \int_{0}^{t}(t-s)^{\alpha-1} M_{2}(s) d s \\
\leq & \left\|x_{0}\right\|_{X}+2 l_{1}+\frac{1}{\Gamma(\alpha)}\left(\int_{0}^{t}(t-s)^{(\alpha-1) /(1-p)} d s\right)^{1-p} \\
& \times\left(\int_{0}^{t} M_{1}^{1 / p}(s) d s\right)^{p}+\frac{1}{\Gamma(\alpha)}
\end{aligned}
$$

$$
\begin{aligned}
& \times\left(\int_{0}^{t}(t-s)^{(\alpha-1) /(1-p)} d s\right)^{1-p}\left(\int_{0}^{t} M_{2}^{1 / p}(s) d s\right)^{p} \\
\leq & \left\|x_{0}\right\|_{X}+2 l_{1}+\frac{c\left(\left\|M_{1}\right\|_{1 / p}+\left\|M_{2}\right\|_{1 / p}\right)}{\Gamma(\alpha)} T^{\alpha-p} \\
= & \lambda_{2} .
\end{aligned}
$$

For $t \in\left(t_{m}, T\right]$,

$$
\begin{aligned}
\|F x(t)\|_{X} \leq & \left\|x_{0}\right\|_{X}+\sum_{k=0}^{m}\left|I_{k}\left(x\left(t_{k}^{-}\right)\right)\right| \\
& +\frac{1}{\Gamma(\alpha)} \int_{0}^{t}(t-s)^{\alpha-1}\|f(s \cdot x(s))\|_{X} d s \\
\leq & \left\|x_{0}\right\|_{X}+m l_{1} \\
& +\frac{1}{\Gamma(\alpha)} \int_{0}^{t}(t-s)^{\alpha-1}\left\|f_{1}(s, x(s))\right\|_{X} d s \\
& +\frac{1}{\Gamma(\alpha)} \int_{0}^{t}(t-s)^{\alpha-1}\left\|f_{2}(s, x(s))\right\| d s \\
\leq & \left\|x_{0}\right\|_{X}+m l_{1}+\frac{1}{\Gamma(\alpha)} \int_{0}^{t}(t-s)^{\alpha-1} M_{1}(s) d s \\
& +\frac{1}{\Gamma(\alpha)} \int_{0}^{t}(t-s)^{\alpha-1} M_{2}(s) d s \\
\leq & \left\|x_{0}\right\|_{X}+m l_{1} \\
& +\frac{1}{\Gamma(\alpha)}\left(\int_{0}^{t}(t-s)^{(\alpha-1) /(1-p)} d s\right)^{1-p} \\
& \times\left(\int_{0}^{t} M_{1}^{1 / p}(s) d s\right)^{p}+\frac{1}{\Gamma(\alpha)} \\
& \times\left(\int_{0}^{t}(t-s)^{(\alpha-1) /(1-p)} d s\right)^{1-p}\left(\int_{0}^{t} M_{2}^{1 / p}(s) d s\right)^{p} \\
\leq & \left\|x_{0}\right\|_{X}+m l_{1}+\frac{c\left(\left\|M_{1}\right\|_{1 / p}+\left\|M_{2}\right\|_{1 / p}\right)}{\Gamma(\alpha)} T^{\alpha-p} \\
= & \lambda_{m},
\end{aligned}
$$

and thus $F\left(B_{\lambda}\right) \subset B_{\lambda}$.

Step 2 ( $F_{1}$ is continuous and $\gamma$-contraction). To prove the continuity of $F_{1}$ for $t \in[0, T]$, let us consider a sequence $x_{n}$ converging to $x$. Taking the norm of $F_{1} x_{n}(t)-F_{1} x(t)$, we have

$$
\begin{aligned}
\| F_{1} x_{n}(t) & -F_{1} x(t) \|_{X} \\
\leq & \sum_{0<t_{k}<t}\left\|I_{1}\left(x_{n}\left(t_{k}^{-}\right)-x\left(t_{k}^{-}\right)\right)\right\|_{X} \\
& +\frac{1}{\Gamma \alpha} \int_{0}^{t}(t-s)^{\alpha-1} L(s)\left\|x_{n}(s)-x(s)\right\|_{X} d s \\
\leq & \sum_{0<t_{k}<t} l_{2} \|\left(x_{n}\left(t_{k}^{-}\right)-x\left(t_{k}^{-}\right) \|_{X}\right.
\end{aligned}
$$




$$
\begin{aligned}
& +\frac{1}{\Gamma \alpha}\left(\int_{0}^{t}(t-s)^{(\alpha-1) /(1-p)} d s\right)^{1-p} \\
& \times\left(\int_{0}^{t} L_{1}^{1 / p}(s) d s\right)^{p}\left\|x_{n}-x\right\| \\
& \leq\left(m l_{2}+\frac{c\left\|L_{1}\right\|_{1 / p} T^{\alpha-p}}{\Gamma \alpha}\right)\left\|x_{n}-x\right\| .
\end{aligned}
$$

From the pervoius analysis, we obtain

$$
\left\|F_{1} x_{n}-F_{1} x\right\| \leq\left(m l_{2}+\frac{c\left\|L_{1}\right\|_{1 / p} T^{\alpha-p}}{\Gamma(\alpha)}\right)\left\|x_{n}-x\right\| .
$$

To prove that $F_{1}$ is $\gamma_{1}$-contraction, let us consider that, for $x, y \in B_{r}$,

$$
\begin{aligned}
\| F_{1} x(t) & -F_{1} y(t) \|_{X} \\
\leq & \sum_{0<t_{k}<t}\left\|I_{k}\left(x\left(t_{k}^{-}\right)-y\left(t_{k}^{-}\right)\right)\right\|_{X} \\
& +\frac{1}{\Gamma(\alpha)} \int_{0}^{t}(t-s)^{\alpha-1} L_{1}(s)\|x(s)-y(s)\|_{X} d s \\
\leq & \sum_{0<t_{k}<t} l_{2} \|\left(x\left(t_{k}^{-}\right)-y\left(t_{k}^{-}\right) \|_{X}\right. \\
& +\frac{1}{\Gamma(\alpha)}\left(\int_{0}^{t}(t-s)^{(\alpha-1) /(1-p)} d s\right)^{1-p} \\
& \times\left(\int_{0}^{t} L_{1}^{1 / p}(s) d s\right)^{p}\|x-y\| \\
\leq & \left(m l_{2}+\frac{c\left\|L_{1}\right\|_{1 / p} T^{\alpha-p}}{\Gamma(\alpha)}\right)\|x-y\| .
\end{aligned}
$$

Thus, for

$$
\gamma_{1}=\left(m l_{2}+\frac{c\left\|L_{1}\right\|_{1 / p} T^{\alpha-p}}{\Gamma(\alpha)}\right)<1,
$$

$F_{1}$ is $\gamma_{1}$-contraction.

Step 3 ( $F_{2}$ is compact). For $0 \leq \tau_{1} \leq \tau_{2} \leq T$, we have

$$
\begin{aligned}
& \left\|F_{2} x\left(\tau_{2}\right)-F_{2} x\left(\tau_{1}\right)\right\|_{X} \\
& \leq \frac{1}{\Gamma(\alpha)} \| \int_{0}^{\tau_{2}}\left(\tau_{2}-s\right)^{\alpha-1} f_{2}(s, x(s)) d s \\
& \quad-\int_{0}^{\tau_{1}}\left(\tau_{1}-s\right)^{\alpha-1} f_{2}(s, x(s)) d s \|_{X} \\
& \leq \frac{1}{\Gamma(\alpha)} \| \int_{0}^{\tau_{1}}\left(\tau_{2}-s\right)^{\alpha-1} f_{2}(s, x(s)) d s \\
& \quad+\int_{\tau_{1}}^{\tau_{2}}\left(\tau_{2}-s\right)^{\alpha-1} f_{2}(s, x(s)) d s \\
& \quad-\int_{0}^{\tau_{1}}\left(\tau_{1}-s\right)^{\alpha-1} f_{2}(s, x(s)) d s \|_{X}
\end{aligned}
$$

$$
\begin{aligned}
& \leq \frac{1}{\Gamma(\alpha)} \int_{0}^{\tau_{1}}\left(\left(\tau_{1}-s\right)^{\alpha-1}-\left(\tau_{2}-s\right)^{\alpha-1}\right) \\
& \quad \times\left\|f_{2}(s, x(s))\right\|_{X} d s \\
& \quad+\frac{1}{\Gamma(\alpha)} \int_{\tau_{1}}^{\tau_{2}}\left(\tau_{2}-s\right)^{\alpha-1}\left\|f_{2}(s, x(s))\right\|_{X} d s \\
& \quad\left((\tau-s)^{\alpha-1} \text { is a decreasing function of } \tau-s .\right)
\end{aligned}
$$$$
\leq \frac{1}{\Gamma(\alpha)} \int_{0}^{\tau_{1}}\left(\left(\tau_{1}-s\right)^{\alpha-1}-\left(\tau_{2}-s\right)^{\alpha-1}\right) M_{2}(s) d s
$$$$
+\frac{1}{\Gamma(\alpha)} \int_{\tau_{1}}^{\tau_{2}}\left(\tau_{2}-s\right)^{\alpha-1} M_{2}(s) d s
$$$$
\leq \frac{1}{\Gamma(\alpha)}\left(\left(\int _ { 0 } ^ { \tau _ { 1 } } \left(\left(\tau_{1}-s\right)^{\alpha-1}\right.\right.\right.
$$$$
\left.\left.\left.-\left(\tau_{2}-s\right)^{\alpha-1}\right)^{1 /(1-p)} d s\right)^{1-p}\right)
$$$$
\times\left(\int_{0}^{\tau_{1}} M_{2}^{1 / p}(s) d s\right)^{p}+\frac{1}{\Gamma(\alpha)}
$$$$
\times\left(\int_{\tau_{1}}^{\tau_{2}}\left(\tau_{2}-s\right)^{(\alpha-1) /(1-p)} d s\right)^{1-p}\left(\int_{0}^{\tau_{1}} M_{2}^{1 / p}(s) d s\right)^{p}
$$$$
\leq \frac{1}{\Gamma(\alpha)}\left(\left(\int _ { 0 } ^ { \tau _ { 1 } } \left(\left(\tau_{1}-s\right)^{(\alpha-1) /(1-p)}\right.\right.\right.
$$$$
\left.\left.\left.-\left(\tau_{2}-s\right)^{(\alpha-1) /(1-p)}\right) d s\right)^{1-p}\right)
$$

$$
\times\left(\int_{0}^{\tau_{1}} M_{2}^{1 / p}(s) d s\right)^{p}
$$$$
\left(x^{z}-y^{z} \geq(x-y)^{z}, \forall x \geq y \geq 0, z>1\right. \text {.) }
$$$$
+\frac{1}{\Gamma(\alpha)}\left(\int_{\tau_{1}}^{\tau_{2}}\left(\tau_{2}-s\right)^{(\alpha-1) /(1-p)} d s\right)^{1-p}
$$$$
\times\left(\int_{0}^{\tau_{1}} M_{2}^{1 / p}(s) d s\right)^{p}
$$$$
\leq \frac{c}{\Gamma(\alpha)}\left(\tau_{1}^{(\alpha-p) /(1-p)}-\tau_{2}^{(\alpha-p) /(1-p)}\right.
$$

$$
\left.+\left(\tau_{2}-\tau_{1}\right)^{(\alpha-p) /(1-p)}\right)^{1-p}
$$

$$
\times\left\|M_{2}\right\|_{1 / p}+\frac{c}{\Gamma(\alpha)}\left(\tau_{2}-\tau_{1}\right)^{\alpha-p}\left\|M_{2}\right\|_{1 / p}
$$

$\leq \frac{c}{\Gamma(\alpha)}\left(\left(\tau_{1}-\tau_{2}\right)^{(\alpha-p) /(1-p)}\right)^{1-p}\left\|M_{2}\right\|_{1 / p}$

$$
+\frac{c}{\Gamma(\alpha)}\left(\tau_{2}-\tau_{1}\right)^{\alpha-p}\left\|M_{2}\right\|_{1 / p} \quad\left(\tau_{2}>\tau_{1} .\right)
$$

$\leq \frac{2 c\left\|M_{2}\right\|_{1 / p}}{\Gamma(\alpha)}\left(\tau_{2}-\tau_{1}\right)^{\alpha-p}$. 
The right-hand side of the pervoiusly expression does not depend on $x$. Thus using Arzela-Ascoli theorem for equicontinuous functions (Diethelm, Theorem D.10 [10]), we conclude that $F_{2}\left(B_{r}\right)$ is relatively compact, and hence $F_{2}$ is completely continuous on $I-\left\{t_{1}, t_{2}, \ldots, t_{m}\right\}$. In a similar way, it can be prove the equi-continuity of $F$ on $t=t_{k}^{-}$and $t=t_{k}^{+}$, $k=1,2, \ldots, m$. And thus $F_{2}$ is compact on $[0, T]$.

Step 4 ( $F$ is condensing). As $F=F_{1}+F_{2}, F_{1}$ is continuous contraction, $F_{2}$ is compact, so by using Lemma $6, F$ is condensing map on $B_{r}$.

And hence by using the Theorem 5, we conclude that (4) has a solution in $B_{r}$.

Theorem 9. If $f$ is bounded and Lipschitz, in particular, $\|f(t, x)-f(t, y)\|_{X} \leq L_{1}^{*}(t)\|x-y\|_{X}$ for all $(t, x),(t, y) \in R$ and $L_{1}^{*} \in L_{1 / p}\left([0, T], \mathbb{R}^{+}\right)$, then the problem (4) has a unique solution in $B_{\lambda}$, provided that

$$
\gamma_{1}^{*}=m l_{2}+\frac{c\left\|L_{1}^{*}\right\|(1 / p) T^{\alpha-p}}{\Gamma(\alpha+1)}<1,
$$

where $c=((1-p) /(\alpha-p))^{1-p}$.

\section{Impulsive Fractional Differential Equations with Finite Delay}

In this section, we discuss existence and uniqueness of solutions of the following impulsive fractional differential equations of Caputo type with finite delay in a Banach space $X$ with norm $|\cdot|$

$$
\begin{gathered}
{ }^{C} D^{\alpha} x(t)=f\left(t, x_{t}\right), \quad t \in I=[0, T], t \neq t_{k}, \\
\left.\Delta x(t)\right|_{t=t_{k}}=I_{k}\left(x\left(t_{k}^{-}\right)\right), \quad k=1,2, \ldots, m, \\
x(t)=\phi(t), \quad t \in[-r, 0],
\end{gathered}
$$

where $f: I \times \mathscr{C} \rightarrow X, \mathscr{C}=C([-r, 0], X)$ with norm $\|x\|_{r}=$ $\sup \left\{\|x(t)\|_{X}: t \in[-r, 0]\right\} . I_{k} \in C(X, X),(k=1,2, \ldots, m)$, and $X$ is a Banach space with a norm $|\cdot|_{X}$. For any $x:[-r, T] \rightarrow$ $X$ and $t \in I, x_{t} \in \mathscr{C}$ and is defined by $x_{t}(s)=x(t+s), s \in$ $[-r, 0]$. Here, our tools are Banach and Schaefer fixed point theorems.

Define a new Banach space $\mathrm{PC}([-r, T], X)$

$$
\begin{aligned}
\mathrm{PC}([-r, T], X)=\left\{x:[-r, T] \longrightarrow X \mid x \in C\left(\left(t_{k}, t_{k+1}\right], X\right)\right. & \cup C([-r, 0], X), k=0,1,2, \ldots, m, \\
& x\left(t_{k}^{+}\right) \text {and } x\left(t_{k}^{-}\right) \text {exist, } \\
& \left.x\left(t_{k}\right)=x\left(t_{k}^{-}\right)\right\}
\end{aligned}
$$

with sup-norm $\|\cdot\|$, defined by $\|x\|=\sup \left\{\|x(t)\|_{X}: t \in\right.$ $[-r, T]\}$.

Definition 10. A solution of fractional differential equation (21) is a piecewise continuous function $x \in \mathrm{PC}([-r, T], X)$ which satisfies (21).
Consider the initial value problem (21) on $I \times \mathscr{C}$ for some fixed $T>0$ and assume that there exist $p \in(0, \alpha), \alpha \in$ $(0,1), M_{3}, M_{4}, L_{2} \in L_{1 / p}\left([0, T], \mathbb{R}^{+}\right)$such that the following assumptions are satisfied:

(A.4) $f \in C(I \times \mathscr{C}, X)$,

(A.5) $f$ bounded, in particular, $\|f(t, \phi)\|_{X} \leq M_{3}(t)$ for all $(t, \phi) \in I \times \mathscr{C}$,

(A.6) $f$ is Lipschitz, in particular, $\|f(t, \phi)-f(t, \psi)\|_{X} \leq$ $L_{2}(t)\|\phi-\psi\|_{r}$ for all $(t, \phi),(t, \psi) \in I \times \mathscr{C}$.

Lemma 11 (Fečken et al. [24], Lemma 2). The initial value problem (21) is equivalent to the nonlinear integral equation

$$
\begin{aligned}
x(t)= & \phi(0)+\frac{1}{\Gamma(\alpha)} \int_{0}^{t}(t-s)^{\alpha-1} f\left(s, x_{s}\right) d s, \\
= & \phi(0)+I_{1}\left(x\left(t_{1}^{-}\right)\right) \\
& +\frac{1}{\Gamma(\alpha)} \int_{0}^{t}(t-s)^{\alpha-1} f\left(s, x_{s}\right) d s, \quad t \in\left(t_{1}, t_{2}\right] \\
= & \phi(0)+\sum_{k=1}^{2} I_{k}\left(x\left(t_{k}^{-}\right)\right) \\
& +\frac{1}{\Gamma(\alpha)} \int_{0}^{t}(t-s)^{\alpha-1} f\left(s, x_{s}\right) d s, \quad t \in\left(t_{2}, t_{3}\right] \\
= & \phi(0)+\sum_{k=1}^{m} I_{k}\left(x\left(t_{k}^{-}\right)\right) \\
& +\frac{1}{\Gamma(\alpha)} \int_{0}^{t}(t-s)^{\alpha-1} f\left(s, x_{s}\right) d s, \quad t \in\left(t_{m}, T\right] \\
= & \phi(t), \quad t \in[-r, 0] .
\end{aligned}
$$

In other words, every solution of the integral equation (23) is also solution of our original initial value problem (21) and conversely.

Remark 12. Since history part/initial condition $x(t)=$ $\phi(t), t \in[-r, 0]$, is known, so we will investigate the existence and uniqueness of solution in $I=[0, T]$.

Theorem 13 (existence and uniqueness of solution). Under the assumptions (A.3)-(A.6), the problem (21) has a unique solution in $[0, T]$, provided that

$$
\gamma_{2}=m l_{2}+\frac{c\left\|L_{2}\right\|_{1 / p} T^{\alpha-p}}{\Gamma(\alpha)}<1,
$$

where $c=((1-p) /(\alpha-p))^{1-p}$.

Proof. In this case, we define the operator $F: \mathrm{PC}(I, X) \rightarrow$ $\operatorname{PC}(I, X)$ by

$$
F x(t)=\sum_{0<t_{k}<t}^{m} I_{k}\left(x_{n}\left(t_{k}^{-}\right)\right)+\frac{1}{\Gamma(\alpha)} \int_{0}^{t}(t-s)^{\alpha-1} f\left(s, x_{s}\right) d s .
$$


Step 1. To prove that $F$ is self mapping, we need to prove that for each $x \in \mathrm{PC}(I, X), F_{x} \in \mathrm{PC}(I, X)$.

The proof is similar to the proof of continuity of $F_{1}$ in Step 2 of Theorem 8, and hence we omit it.

Step 2 ( $F$ is continuous and $\gamma_{2}$-contraction). The proof of this step is also similar to the proof of continuous and $\gamma_{1}$ contraction of $F_{1}$ in Step 2 of Theorem 8 .

Now by applying Banach's fixed point theorem, we get that the operator $F$ has an unique fixed point in $\operatorname{PC}(I, X)$, and hence the problem (21) has a unique solution in $\mathrm{PC}([-r, T], X)$.

Our next result is based on Schaefer's fixed point theorem. In this case, we replace assumption (A.3) with the following linear growth condition:

(A.3') $I_{k}$ bounded, in particular, $\left\|I_{k}(x)\right\|_{X} \leq l_{1}^{*}$,

$\left(\right.$ A. $\left.5^{\prime}\right) f$ bounded, in particular, $\|f(t, \phi)\|_{X} \leq M_{4}(t)(1+$ $\left.\|\phi\|_{r}\right)$ for all $(t, \phi) \in I \times \mathscr{C}$.

Theorem 14. Under the assumptions $\left(A .3^{\prime}\right)$ and $\left(A .5^{\prime}\right)$, problem (21) has at least one solution.

Proof. We transform the problem into a fixed point problem. For this purpose, consider the operator $F: \operatorname{PC}(I, X) \rightarrow$ $\mathrm{PC}(I, X)$ defined by

$$
F x(t)=\sum_{0<t_{k}<t}^{m} I_{k}\left(x_{n}\left(t_{k}^{-}\right)\right)+\frac{1}{\Gamma(\alpha)} \int_{0}^{t}(t-s)^{\alpha-1} f\left(s, x_{s}\right) d s .
$$

Step 1 ( $F$ is continuous). Let $\left\{x^{n}\right\}$ be a sequence such that $x^{n} \rightarrow x$ in $\operatorname{PC}(I, X)$. Then, for every $t \in I$, we have

$$
\begin{aligned}
\| F x^{n}(t) & -F x(t) \|_{X} \\
\leq & \sum_{0<t_{k}<t}\left\|I_{k}\left(x^{n}\left(t_{k}^{-}\right)\right)-I_{k}\left(x\left(t_{k}^{-}\right)\right)\right\| \\
& +\frac{1}{\Gamma \alpha} \int_{0}^{t}(t-s)^{\alpha-1}\left\|f\left(s, x_{s}^{n}\right)-f\left(s, x_{s}\right)\right\|_{X} d s \\
\leq & \sum_{0<t_{k}<t}\left\|I_{k}\left(x^{n}\left(t_{k}^{-}\right)\right)-I_{k}\left(x\left(t_{k}^{-}\right)\right)\right\|_{X} \\
& +\left\|f\left(\cdot, x^{n}\right)-f(\cdot, x .)\right\| \frac{1}{\Gamma \alpha}\left(\int_{0}^{t}(t-s)^{\alpha-1}\right)^{1-p} d s \\
\leq & \sum_{k=1}^{m}\left\|I_{k}\left(x^{n}(\cdot)\right)-I_{k}(x(\cdot))\right\| \\
& +\frac{T^{\alpha}}{\Gamma(\alpha+1)}\left\|f\left(\cdot, x_{.}^{n}\right)-f(\cdot, x .)\right\| .
\end{aligned}
$$

We can see that if $n \rightarrow \infty, F x^{n} \rightarrow F x$, as $x^{n} \rightarrow x$ and $I_{k}$ and both $f$ are continuous. Hence, $F$ is continuous.

Step 2 ( $F$ maps bounded sets into bounded sets in $\mathrm{PC}(I, X)$ ). It is enough to show that for any $\delta>0$, there exists an $l=\|\phi(0)\|_{r}+m l_{1}^{*}+\left(c(1+\delta)\left(\left\|M_{4}\right\|_{1 / p}\right) / \Gamma(\alpha)\right) T^{\alpha-p}>0$ such that $x \in B_{\delta}=\{x \in \operatorname{PC}(I, X) \mid\|x\| \leq \delta\}$, and we have $\|F x\| \leq l$.

For $t \in[0, T]$, we have

$$
\begin{aligned}
\|F x(t)\|_{X} \leq & \|\phi(0)\|_{r}+\sum_{0<t_{k}<t}\left|I_{k}\left(x\left(t_{k}^{-}\right)\right)\right| \\
& +\frac{1}{\Gamma(\alpha)} \int_{0}^{t}(t-s)^{\alpha-1}\left\|f\left(s, x_{s}\right)\right\|_{X} d s \\
\leq & \|\phi(0)\|_{r}+m l_{1}^{*} \\
& +\frac{1}{\Gamma(\alpha)} \int_{0}^{t}(t-s)^{\alpha-1} M_{4}(s)\left(1+\left\|x_{s}\right\|_{r}\right) d s \\
\leq & \|\phi(0)\|_{r}+\frac{(1+\|x\|)}{\Gamma(\alpha)} \int_{0}^{t}(t-s)^{\alpha-1} M_{4}(s) d s \\
\leq & \|\phi(0)\|_{r}+m l_{1}^{*}+\frac{(1+\|x\|)}{\Gamma(\alpha)} \\
& \times\left(\int_{0}^{t}(t-s)^{(\alpha-1) /(1-p)} d s\right)^{1-p}\left(\int_{0}^{t} M_{4}^{1 / p}(s) d s\right)^{p} \\
\leq & \|\phi(0)\|_{r}+m l_{1}^{*}+\frac{c(1+\delta)\left(\left\|M_{4}\right\|_{1 / p}\right)}{\Gamma(\alpha)} T^{\alpha-p} .
\end{aligned}
$$

Step 3 ( $F$ maps bounded sets into equicontinuous sets in $\mathrm{PC}(I, X))$. The proof of this step is similar to the proof of compactness of $F_{2}$ in Step 3 of Theorem 8.

As a consequence of Steps 1-3 together with PC-type Arzela-Ascoli theorem (Fečken et al. [24], Theorem 2.11), the map $F: \mathrm{PC}(I, X) \rightarrow \mathrm{PC}(I, X)$ is completely continuous.

Step 4 (A priori bounds). Now, we prove that the set

$$
E(F)=\{x \in \mathrm{PC}(I, X) \mid x=\lambda F x \text {, for some } \lambda \in(0,1)\}
$$
is bounded.

We observe that for $t \in[0, T]$ and $x \in E(F), x(t)=$ $\lambda F x(t)$ and

$$
\begin{aligned}
\|x(t)\|_{X} \leq & \|F x(t)\|_{X} \\
\leq & \|\phi(0)\|_{r}+\sum_{0<t_{k}<t}\left\|I_{k}\left(x\left(t_{k}^{-}\right)\right)\right\|_{X} \\
& +\frac{1}{\Gamma(\alpha)} \int_{0}^{t}(t-s)^{\alpha-1}\left\|f\left(s, x_{s}\right)\right\|_{X} d s \\
\leq & \|\phi(0)\|_{r}+m l_{1}^{*} \\
& +\frac{1}{\Gamma(\alpha)} \int_{0}^{t}(t-s)^{\alpha-1} M_{4}(s)\left(1+\left\|x_{s}\right\|\right) d s \\
\leq & \|\phi(0)\|_{r}+m l_{1}^{*}+\frac{(1+\|x\|)}{\Gamma(\alpha)}
\end{aligned}
$$




$$
\begin{aligned}
& \times\left(\int_{0}^{t}(t-s)^{(\alpha-1) /(1-p)} d s\right)^{1-p}\left(\int_{0}^{t} M_{4}^{1 / p}(s) d s\right)^{p} \\
\leq & \|\phi(0)\|_{r}+m l_{1}^{*}+\frac{c(1+\|x\|)\left(\left\|M_{4}\right\|_{1 / p}\right)}{\Gamma(\alpha)} T^{\alpha-p},
\end{aligned}
$$

and hence

$$
\|x\| \leq \frac{\|\phi(0)\|_{r}+m l_{1}^{*}+\left(c\left(\left\|M_{4}\right\|_{1 / p}\right) / \Gamma(\alpha)\right) T^{\alpha-p}}{1-\left(c\left(\left\|M_{4}\right\|_{1 / p}\right) / \Gamma(\alpha)\right) T^{\alpha-p}} .
$$

This shows that $E(F)$ is bounded.

As a consequence of Schaefer's fixed point theorem, the problem (21) has at least one solution in $\mathrm{PC}([-r, T], X)$.

Theorem 15. If $f$ is bounded and Lipschitz, in particular, $\|f(t, \phi)-f(t, \psi)\|_{X} \leq L_{2}^{*}(t)\|\phi-\psi\|_{r}$, for all $(t, \phi),(t, \psi) \in I \times \mathscr{C}$ and $L_{2}^{*} \in L_{1 / p}\left([0, T], \mathbb{R}^{+}\right)$, then problem (21) has a unique solution in $P C([-r, T], X)$, provided that

$$
\gamma_{2}^{*}=m l_{2}+\frac{c\left\|L_{2}^{*}\right\|_{1 / p} T^{\alpha-p}}{\Gamma(\alpha+1)}<1,
$$

where $c=((1-p) /(\alpha-p))^{1-p}$.

Further, we consider the following more general Caputo fractional differential equation

$$
\begin{gathered}
{ }^{C} D^{\alpha} x(t)=f\left(t, x(t), x_{t}\right), \quad t \in[0, T], \quad t \neq t_{k}, \\
\left.\Delta x(t)\right|_{t=t_{k}}=I_{k}\left(x\left(t_{k}^{-}\right)\right), \quad k=1,2, \ldots, m, \\
x(t)=\phi(t), \quad t \in[-r, 0],
\end{gathered}
$$

where $f: I \times X \times \mathscr{C} \rightarrow X$ and $\mathscr{C}=C([-r, 0], X)$ with norm $\|x\|_{r}=\sup \left\{\|x(t)\|_{X}: t \in[-r, 0]\right\}, I_{k} \in C(X, X),(k=$ $1,2, \ldots, m)$, and $X$ is a separable real Banach space with the norm $\|\cdot\|_{X}$. Here, our tools will be Banach and Schaefer fixed point theorems.

Definition 16. A solution of fractional differential equation (32) is a piecewise continuous function $x \in \mathrm{PC}([-r, T], X)$ which satisfies (32).

Consider the initial value problem (32) on $I \times \mathscr{C} \times X$ for some fixed $T>0$ and assume that there exist $p \in(0, \alpha)$, $M_{5}, M_{6}, L_{3}, L_{4} \in L_{1 / p}\left([0, T], \mathbb{R}^{+}\right)$such that the following assumptions are satisfied:

(A.7) $f \in C(I \times X \times \mathscr{C}, X)$,

(A.8) $f$ bounded, in particular, $\|f(t, x, \phi)\|_{X} \leq M_{5}(t)$ for all $(t, x, \phi) \in I \times X \times \mathscr{C}$,

(A.9) $f$ is Lipschitz, in particular, $\| f(t, x, \phi) \quad-$ $f(t, y, \psi)\left\|_{X} \leq L_{3}(t)\right\| x-y\left\|_{X}+L_{4}(t)\right\| \phi-\psi \|_{r}$ for all $(t, x, \phi),(t, y, \psi) \in I \times X \times \mathscr{C}$,

$\left(\mathrm{A} .8^{\prime}\right)\|f(t, x, \phi)\|_{X} \leq M_{6}(t)\left(1+\|x\|_{X}+\|\phi\|_{r}\right)$.
Lemma 17 (Fečken et al. [24], Lemma 2). The initial value problem (32) is equivalent to the following nonlinear integral equation

$$
\begin{aligned}
x(t)= & \phi(0)+\frac{1}{\Gamma(\alpha)} \int_{0}^{t}(t-s)^{\alpha-1} f\left(s, x(s), x_{s}\right) d s \\
= & \phi(0)+I_{1}\left(x\left(t_{1}^{-}\right)\right) \\
& +\frac{1}{\Gamma(\alpha)} \int_{0}^{t}(t-s)^{\alpha-1} f\left(s, x(s), x_{s}\right) d s, \quad t \in\left(t_{1}, t_{2}\right] \\
= & \phi(0)+\sum_{k=1}^{2} I_{k}\left(x\left(t_{k}^{-}\right)\right) \\
& +\frac{1}{\Gamma(\alpha)} \int_{0}^{t}(t-s)^{\alpha-1} f\left(s, x(s), x_{s}\right) d s, \quad t \in\left(t_{2}, t_{3}\right] \\
= & \phi(0)+\sum_{k=1}^{m} I_{k}\left(x\left(t_{k}^{-}\right)\right) \\
& +\frac{1}{\Gamma(\alpha)} \int_{0}^{t}(t-s)^{\alpha-1} f\left(s, x(s), x_{s}\right) d s, \quad t \in\left(t_{m}, T\right] \\
= & \phi(t), \quad t \in[-r, 0] .
\end{aligned}
$$

In other words, every solution of the integral equation (33) is also solution of our original initial value problem (32) and conversely.

Theorem 18. Under the assumptions (A.3), (A.7), (A.8), and (A.9), the problem (32) has a unique solution, provided that

$$
\gamma_{3}=m l_{2}+\frac{c\left(\left\|L_{3}\right\|_{1 / p}+\left\|L_{4}\right\|_{1 / p}\right) T^{\alpha-p}}{\Gamma(\alpha)}<1 .
$$

Proof. The proof is similar to the proof of Theorem 13.

Theorem 19. Under the assumptions $\left(A .3^{\prime}\right)$, and $\left(A .8^{\prime}\right)$, the problem (32) has at least one solution in $P C([-r, T], X)$.

Proof. The proof is similar to the proof of Theorem 14 .

Theorem 20. If $f$ is bounded and Lipschitz, in particular, $\|f(t, x, \phi)-f(t, y, \psi)\|_{X} \leq L_{3}^{*}(t)\|x-y\|_{X}+L_{4}^{*}(t)\|\phi-\psi\|_{r}$, for all $(t, x, \phi),(t, y, \psi) \in I \times X \times \mathscr{C}$ and $L_{3}^{*}, L_{4}^{*} \in L_{1 / p}\left([0, T], \mathbb{R}^{+}\right)$, then the problem (32) has a unique solution in $P C([-r, T], X)$, provided that

$$
\gamma_{3}^{*}=m l_{2}+\frac{c\left(\left\|L_{3}^{*}\right\|_{1 / p}+\left\|L_{2}^{*}\right\|_{1 / p}\right) T^{\alpha-p}}{\Gamma(\alpha+1)}<1,
$$

where $c=((1-p) /(\alpha-p))^{1-p}$. 


\section{Examples}

Example 21 (fractional impulsive logistic equation). Consider the following class of fractional logistic equations in banach space $X$ with norm $\|\cdot\|_{X}$ :

$$
\begin{gathered}
{ }^{C} D^{\alpha} x(t)=x(t)(a(t)-b(t) x(t)), \quad t \in[0, T], t \neq t_{k}, \\
\left.\Delta x(t)\right|_{t=t_{k}}=I_{k}\left(x\left(t_{k}^{-}\right)\right), \quad k=1,2, \ldots, m, \\
x(0)=x_{0},
\end{gathered}
$$

where $a(t) \in\left[a_{*}, a^{*}\right]$ and $b(t) \in\left[b_{*}, b^{*}\right]$ with $a_{*}, b_{*}>0$.

Lemma 22 (Fečken et al. [24], Lemma 2). The initial value problem (36) is equivalent to the nonlinear integral equation

$$
\begin{aligned}
& x(t)= x_{0}+\frac{1}{\Gamma(\alpha)} \int_{0}^{t}(t-s)^{\alpha-1}(x(s)(a(s)-b(s) x(s))) d s \\
& t \in\left[0, t_{1}\right] \\
&= x_{0}+I_{1}\left(x\left(t_{1}^{-}\right)\right)+\frac{1}{\Gamma(\alpha)} \int_{0}^{t}(t-s)^{\alpha-1} x(s) a(s) d s \\
&-\frac{1}{\Gamma(\alpha)} \int_{0}^{t}(t-s)^{\alpha-1} b(s) x^{2}(s) d s, \quad t \in\left(t_{1}, t_{2}\right] \\
&= x_{0}+\sum_{k=1}^{2} I_{k}\left(x\left(t_{k}^{-}\right)\right)+\frac{1}{\Gamma(\alpha)} \int_{0}^{t}(t-s)^{\alpha-1} x(s) a(s) d s \\
&-\frac{1}{\Gamma(\alpha)} \int_{0}^{t}(t-s)^{\alpha-1} b(s) x^{2}(s) d s, \\
&= x_{0}+\sum_{k=1}^{m} I_{k}\left(x\left(t_{k}^{-}\right)\right)+\frac{1}{\Gamma(\alpha)} \int_{0}^{t}(t-s)^{\alpha-1} x(s) a(s) d s \\
&-\frac{1}{\Gamma(\alpha)} \int_{0}^{t}(t-s)^{\alpha-1} b(s) x^{2}(s) d s, \quad t \in\left[t_{m}, T\right] .
\end{aligned}
$$

In other words, every solution of the integral equation (37) is also solution of our original initial value problem (36) and conversely.

We can easily see that for the problem (36), our functions are $f_{1}(t, x)=a(t) x$ and $f_{2}(t, x)=-b(t) x^{2}$. It is not difficult to deduce that

$$
\begin{gathered}
\left\|f_{1}(t, x)\right\|_{X} \leq a^{*}\|x\|_{X}+m l_{1}, \\
\left\|f_{1}(t, x)-f_{1}(t, y)\right\|_{X} \leq a^{*}\|x-y\|_{X} .
\end{gathered}
$$

Also $\left\|f_{2}(t, x)\right\|_{X} \leq b^{*}\|x\|^{2}$. From the integral representation of problem (36), we get

$$
\|x(t)\|_{X} \leq\left\|x_{0}\right\|_{X}+m l_{1}+\frac{1}{\Gamma(\alpha)} \int_{0}^{t}(t-s)^{\alpha-1} a^{*}\|x(s)\|_{X} d s .
$$

Using Gronwall's inequality (Diethelm, Lemma 6.19 [10, 31]), we get

$$
\begin{aligned}
\|x(t)\|_{X} & \leq\left(\left\|x_{0}\right\|_{X}+m l_{1}\right) \exp \left(\frac{1}{\Gamma(\alpha)} \int_{0}^{t}(t-s)^{\alpha-1} a^{*} d s\right) \\
& \leq\left(\left\|x_{0}\right\|_{X}+m l_{1}\right) \exp \left(\frac{a^{*}}{\Gamma(\alpha+1)}\right) .
\end{aligned}
$$

Thus $x$ is bounded which implies that all the assumptions of Theorem 8 are satisfied, and hence there exists a solution of the problem (36).

We give some more examples which are inspired by [24].

Example 23. Consider the following Caputo impulsive delay fractional differential equations

$$
\begin{gathered}
{ }^{C} D^{\alpha} x(t)=\frac{e^{-v t}\left\|x_{t}\right\|}{\left(1+e^{t}\right)\left(1+\left\|x_{t}\right\|\right)}, \quad t \in[0,1], t \neq t_{1}, v>0, \\
\left.\Delta x(t)\right|_{t=t_{1}}=\frac{1}{2}, \\
x(t)=\phi(t), \quad t \in[-r, 0] .
\end{gathered}
$$

Set $C_{1}=C\left([-r, 0], \mathbb{R}^{+}\right), f(t, \phi)=e^{-v t} \phi /\left(\left(1+e^{t}\right)(1+\right.$ $\phi)),(t, \phi) \in[0,1] \times C_{1}$.

Let $\phi_{1}, \phi_{2} \in C_{1}$ and let $t \in[0,1]$. Then, we have

$$
\begin{aligned}
\left|f\left(t, \phi_{1}\right)-f\left(t, \phi_{2}\right)\right| & =\frac{e^{-v t}}{1+e^{t}}\left|\frac{\phi_{1}}{1+\phi_{1}}-\frac{\phi_{2}}{1+\phi_{2}}\right| \\
& =\frac{e^{-v t}\left\|\phi_{1}-\phi_{2}\right\|_{r}}{\left(1+e^{t}\right)\left(1+\phi_{1}\right)\left(1+\phi_{2}\right)} \\
& \leq \frac{e^{-v t}\left\|\phi_{1}-\phi_{2}\right\|_{r}}{\left(1+e^{t}\right)} \leq L^{*}(t)\left\|\phi_{1}-\phi_{2}\right\|_{r},
\end{aligned}
$$

where $L^{*}(t)=e^{-v t} / 2$.

Again, for all $\phi \in C_{1}$ and each $t \in[0, T]$,

$$
\begin{aligned}
|f(t, \phi)| & =\frac{e^{-v t}}{1+e^{t}}\left|\frac{\phi}{1+\phi}\right| \leq \frac{e^{-v t}}{\left(1+e^{t}\right)} \\
& <m_{1}(t),
\end{aligned}
$$

where $m_{1}(t)=e^{-v t} / 2$.

For $t \in[0,1]$ and some $p \in(0, \alpha), L^{*}(t)=m_{1}(t)=$ $e^{-v t} / 2 \in L_{1 / p}\left([0,1], \mathbb{R}^{+}\right)$with $M_{1}^{*}=\left\|e^{-v t} / 10\right\|_{1 / p}$, we can arrive at the inequality $1 / 4+c M_{1}^{*} / \Gamma(\alpha)<1$. We can see that all the assumptions of Theorem 13 are satisfied, and hence the problem (41) has a unique solution in $[0,1]$. 
Example 24. Consider the following Caputo impulsive delay fractional differential equation

$$
\begin{gathered}
{ }^{C} D^{\alpha} x(t)=\frac{\left\|x_{t}\right\|}{\left(1+e^{t}\right)\left(1+\left\|x_{t}\right\|\right)}, \quad t \in[0,1], t \neq t_{1}, v>0, \\
\left.\Delta x(t)\right|_{t=t_{1}}=\frac{1}{2}, \\
x(t)=\phi(t), \quad t \in[-r, 0] .
\end{gathered}
$$

Set $C_{2}=C\left([0,1], \mathbb{R}^{+}\right), f(t, \phi)=\phi /\left(\left(1+e^{t}\right)(1+\right.$ $\phi)),(t, \phi) \in[-r, 0] \times C_{2}$.

Again, for all $\phi \in C_{2}$ and each $t \in[0, T]$,

$$
\begin{aligned}
|f(t, \phi)| & =\frac{e^{-t}}{1+e^{t}}\left|\frac{\phi}{1+\phi}\right| \\
& \leq \frac{e^{-t}}{\left(1+e^{t}\right)}<m_{2}(t)(1+\|\phi\|),
\end{aligned}
$$

where $m_{2}(t)=e^{-t} / 4$.

For $t \in[0,1]$ and some $p \in(0, \alpha), m_{2}(t)=e^{-t} / 4 \epsilon$ $L_{1 / p}\left([0,1], \mathbb{R}^{+}\right)$with $M_{2}^{*}=\left\|e^{-t} / 4\right\|_{1 / p}$, we can arrive at the inequality $1 / 4+c M / \Gamma(\alpha)<1$. We can see that all the assumptions of Theorem 14 are satisfied, and hence the problem (44) has a solution in $[0,1]$.

\section{Acknowledgments}

The authors are thankful to the anonymous reviewer for his/her valuable comments and suggestions. S. Abbas acknowledge "Erasmus Mundus Lot13, India 4EU" for providing him fellowship to visit University of Bologna, Italy. He also acknowledges Professor Stefan Siegmund, Center for Dynamics of TU Dresden, Germany, for his fruitful discussion on this topic and for hosting his short visit.

\section{References}

[1] V. D. Milman and A. D. Myškis, "On the stability of motion in the presence of impulses," Siberial Mathematical Journal, vol. 1, pp. 233-237, 1960.

[2] A. M. Samoilenko and N. A. Perestyuk, Differential Equations With Impulses, Viska Scola, Kiev, Ukraine, 1987.

[3] V. Lakshmikantham, D. D. Baĭnov, and P. S. Simeonov, Theory of Impulsive Differential Equations, vol. 6 of Series in Modern Applied Mathematics, World Scientific, Teaneck, NJ, USA, 1989.

[4] D. D. Bainov and P. S. Simeonov, Systems with Impulsive Effects, Horwood, Chichister, UK, 1989.

[5] D. D. Bainov and P. S. Simeonov, Impulsive DiffErential Equations: Periodic Solutions and Its Applications, Longman Scientific and Technical Group, Harlow, UK, 1993.

[6] D. Bainov and V. Covachev, Impulsive Differential Equations with a Small Parameter, vol. 24 of Series on Advances in Mathematics for Applied Sciences, World Scientific, River Edge, NJ, USA, 1994.

[7] M. Benchohra, J. Henderson, and S. K. Ntonyas, Impulsive Diffrential Equations and Inclusions, vol. 2, Hindawi Publishing Corporation, New York, NY, USA, 2006.
[8] J. Hale, Theory of Functional Differential Equations, Springer, New York, NY, USA, 2nd edition, 1977.

[9] F. Mainardi, "Fractional calculus: some basic problems in continuum and statistical mechanics," in Fractals and Fractional Calculus in Continuum Mechanics, vol. 378 of CISM Courses and Lectures, pp. 291-348, Springer, Vienna, Austria, 1997.

[10] K. Diethelm, The Analysis of Fractional Differential Equations, vol. 2004 of Lecture Notes in Mathematics, Springer, Berlin, Germany, 2010.

[11] A. A. Kilbas, H. M. Srivastava, and J. J. Trujillo, Theory and Applications of Fractional Differential Equations, vol. 204, North-Holland Mathematics Studies, 2006.

[12] K. S. Miller and B. Ross, An Introduction to the Fractional Calculus and Fractional Differential Equations, A Wiley-Interscience Publication, John Wiley \& Sons, New York, NY, USA, 1993.

[13] I. Podlubny, Fractional Differential Equations, vol. 198 of Mathematics in Science and Engineering, Academic Press, San Diego, Calif, USA, 1999.

[14] J. Sabatier, O. P. Agrawal, and J. A. Tenreiro Machado, Advances in Fractional Calculus, Springer, Dordrecht, The Netherlands, 2007.

[15] S. G. Samko, A. A. Kilbas, and O. I. Marichev, Fractional Integrals and Derivatives, Gordon and Breach Science, Yverdon, Switzerland, 1993.

[16] S. Abbas, "Existence of solutions to fractional order ordinary and delay differential equations and applications," Electronic Journal of Differential Equations, vol. 2011, no. 9, pp. 1-11, 2011.

[17] S. Abbas, M. Banerjee, and S. Momani, "Dynamical analysis of fractional-order modified logistic model," Computers \& Mathematics with Applications, vol. 62, no. 3, pp. 1098-1104, 2011.

[18] E. Ahmed, A. M. A. El-Sayed, and H. A. A. El-Saka, "Equilibrium points, stability and numerical solutions of fractionalorder predator-prey and rabies models," Journal of Mathematical Analysis and Applications, vol. 325, no. 1, pp. 542-553, 2007.

[19] S. B. Hadid, "Local and global existence theorems on differential equations of non-integer order," Journal of Fractional Calculus, vol. 7, pp. 101-105, 1995.

[20] R. W. Ibrahim and S. Momani, "On the existence and uniqueness of solutions of a class of fractional differential equations," Journal of Mathematical Analysis and Applications, vol. 334, no. 1, pp. 1-10, 2007.

[21] V. Lakshmikantham and A. S. Vatsala, "Basic theory of fractional differential equations," Nonlinear Analysis: Theory, Methods \& Applications, vol. 69, no. 8, pp. 2677-2682, 2008.

[22] R. P. Agarwal, M. Benchohra, and B. A. Slimani, "Existence results for differential equations with fractional order and impulses," Georgian Academy of Sciences. A. Razmadze Mathematical Institute. Memoirs on Differential Equations and Mathematical Physics, vol. 44, pp. 1-21, 2008.

[23] M. Benchohra and B. A. Slimani, "Existence and uniqueness of solutions to impulsive fractional differential equations," Electronic Journal of Differential Equations, vol. 2009, no. 10, pp. 1-11, 2009.

[24] M. Fečkan, Y. Zhou, and J. Wang, "On the concept and existence of solution for impulsive fractional differential equations," Communications in Nonlinear Science and Numerical Simulation, vol. 17, no. 7, pp. 3050-3060, 2012.

[25] D. Xu, Y. Hueng, and L. Ling, "Existence of positive solutions of an Impulsive Delay Fishing model," Bulletin of Mathematical Analysis and Applications, vol. 3, no. 2, pp. 89-94, 2011. 
[26] C. Giannantoni, "The problem of the initial conditions and their physical meaning in linear differential equations of fractional order," Applied Mathematics and Computation, vol. 141, no. 1, pp. 87-102, 2003.

[27] N. Heymans and I. Podlubny, "Physical interpretation of initial conditions for fractional differential equations with RiemannLiouville fractional derivatives," Rheologica Acta, vol. 45, no. 5, pp. 765-771, 2006.

[28] I. Podlubny, "Geometric and physical interpretation of fractional integration and fractional differentiation," Fractional Calculus \& Applied Analysis, vol. 5, no. 4, pp. 367-386, 2002, Dedicated to the 60th anniversary of Prof. Francesco Mainard.

[29] E. Zeidler, Non-Linear Functional Analysis and Its Application: Fixed Point-Theorems, vol. 1, Springer, New York, NY, USA, 1986.

[30] B. N. Sadovskiı̌, "On a fixed point principle," Functional Analysis and Its Applications, vol. 1, no. 2, pp. 74-76, 1967.

[31] H. Ye, J. Gao, and Y. Ding, "A generalized Gronwall inequality and its application to a fractional differential equation," Journal of Mathematical Analysis and Applications, vol. 328, no. 2, pp. 1075-1081, 2007. 


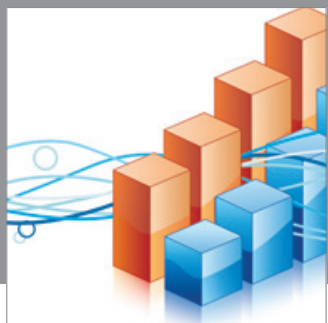

Advances in

Operations Research

mansans

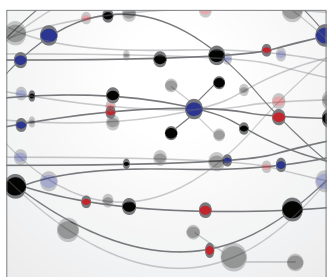

The Scientific World Journal
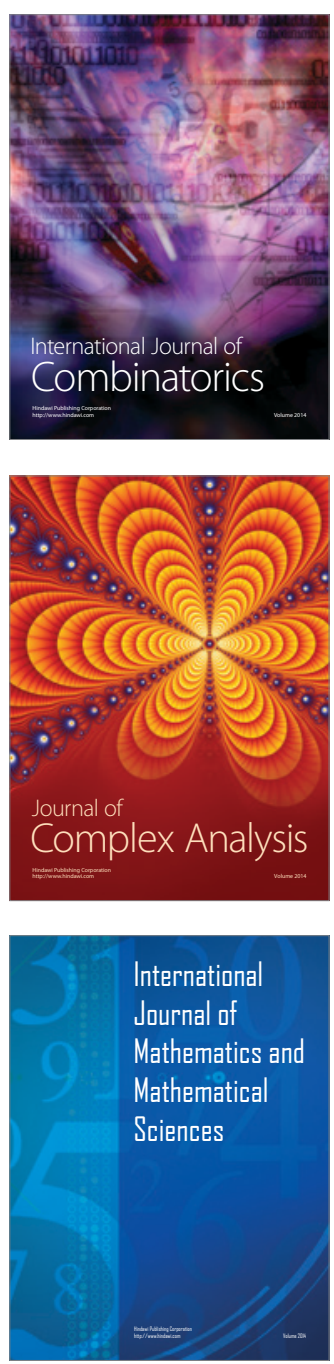
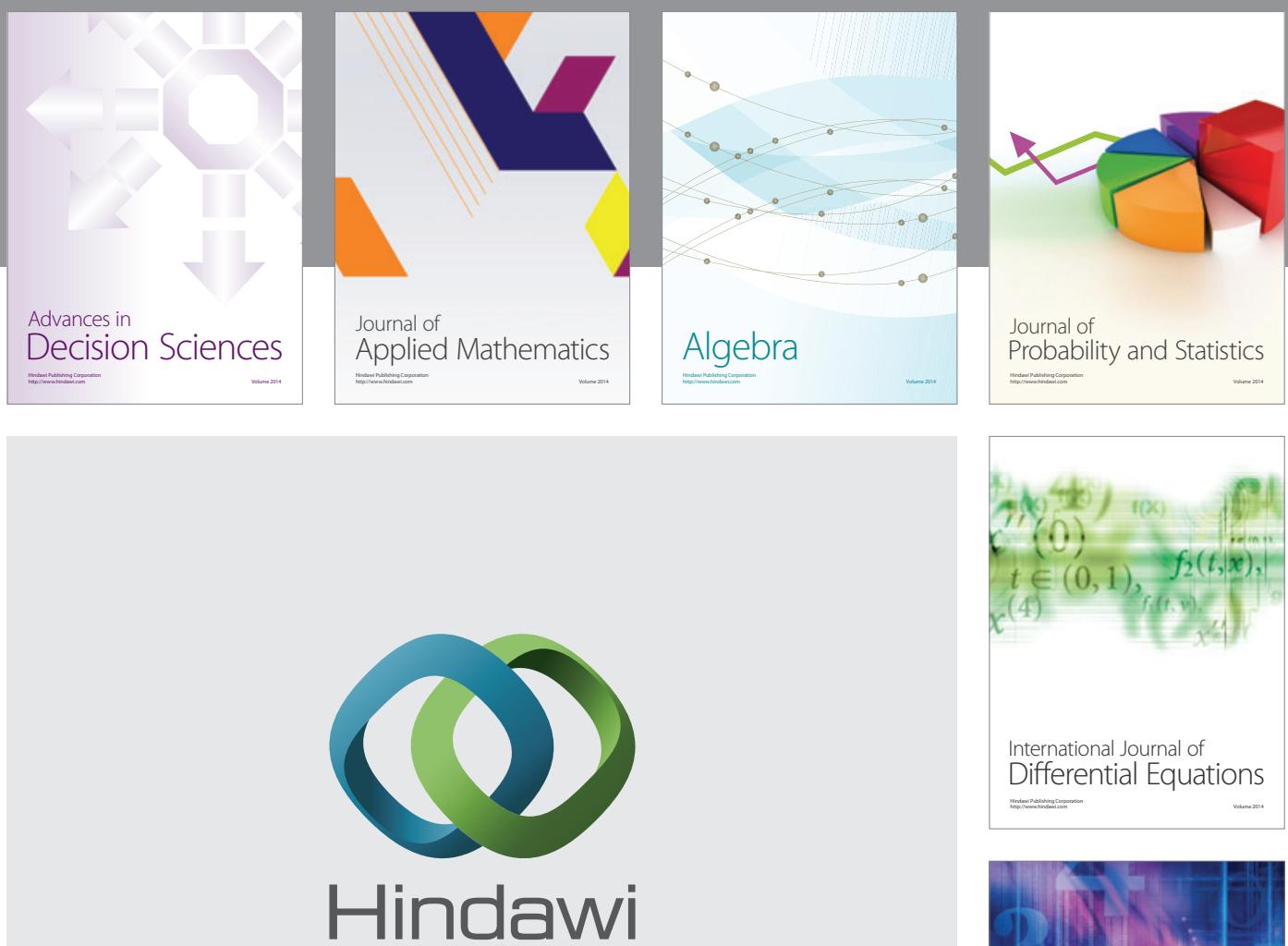

Submit your manuscripts at http://www.hindawi.com
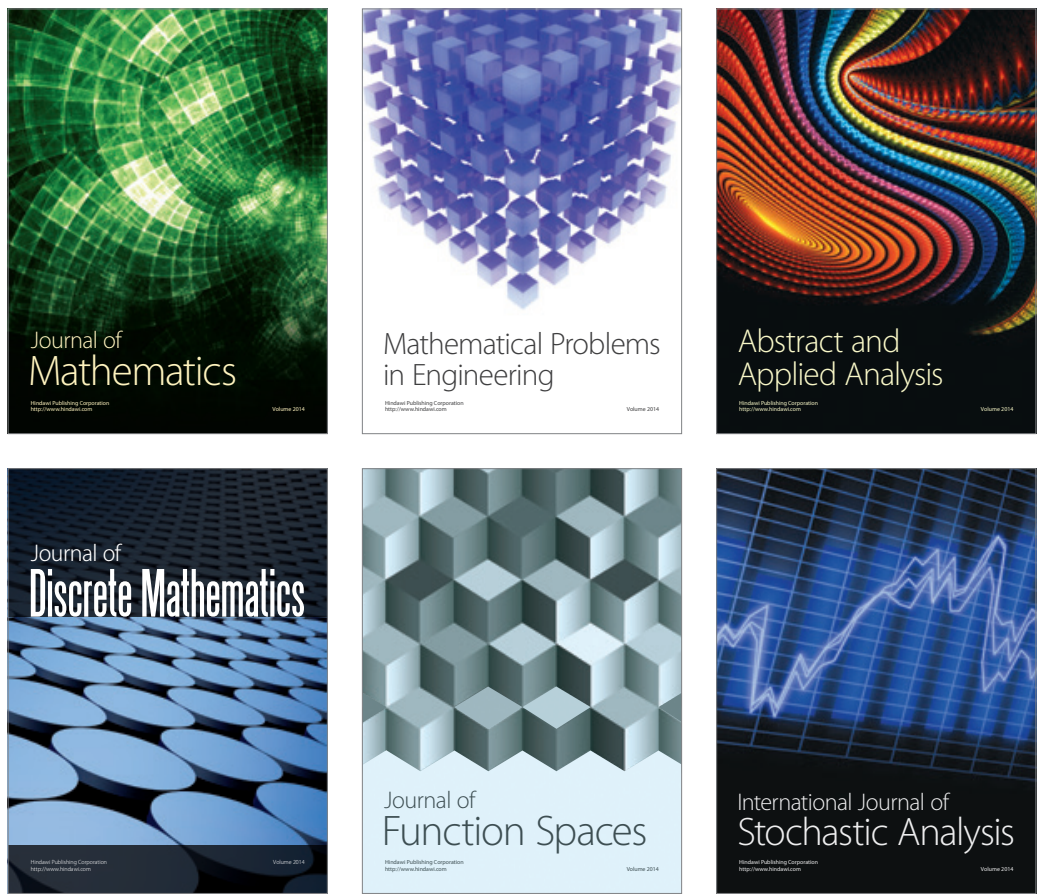

Journal of

Function Spaces

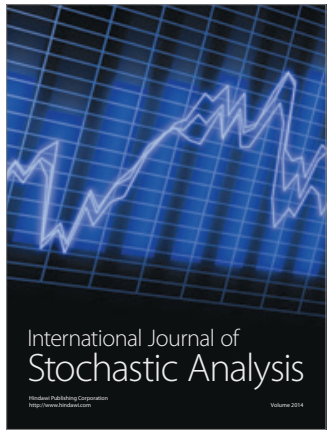

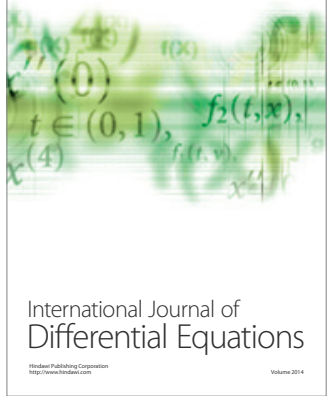
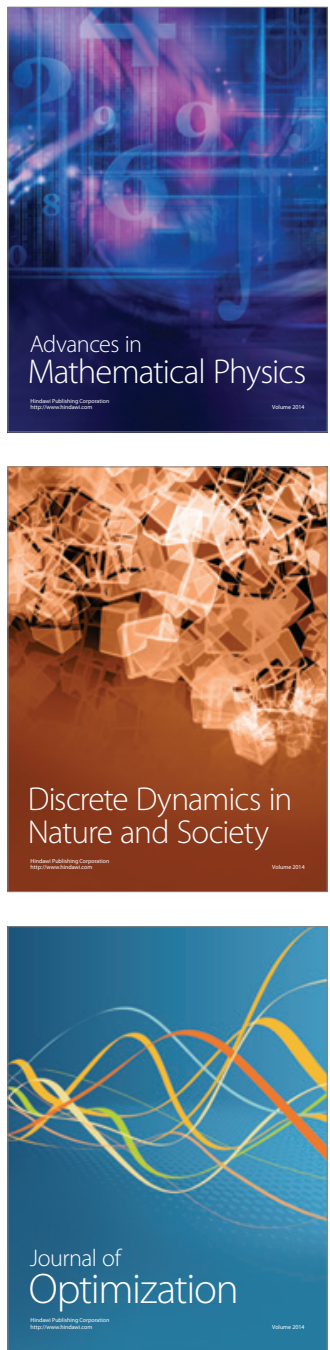